\title{
Stereoselective synthesis of four possible isomers of streptopyrrolidine
}

\author{
Debendra K. Mohapatra*, Barla Thirupathi, Pragna P. Das \\ and Jhillu S. Yadav*
}

\section{Full Research Paper}

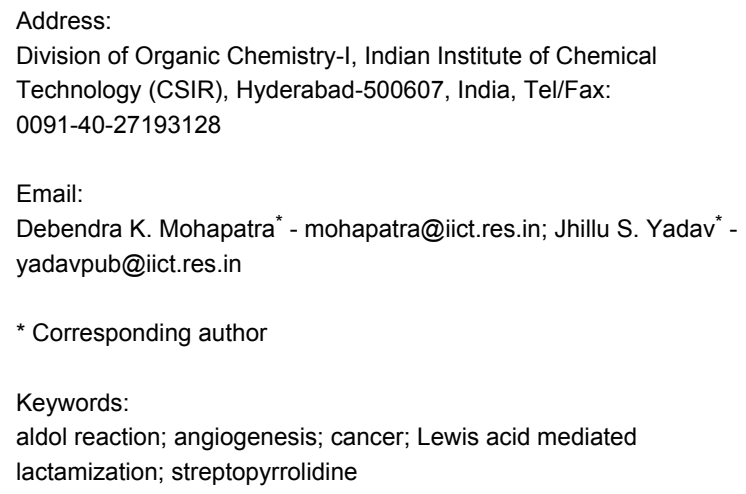

\section{Abstract}

The synthesis of $(4 R, 5 R)$-streptopyrrolidine (1), (4S,5R)-streptopyrrolidine (2) (4R,5S)-streptopyrrolidine (3) and (4S,5S)-streptopyrrolidine (4) have been achieved in a concise and highly efficient manner via a highly stereoselective aldol type reaction with the trimethylsilyl enolate of ethyl acetate and Lewis acid mediated lactamization as the key reactions in $\approx 42 \%$ yield over six steps starting from D-phenylalanine and L-phenylalanine, respectively. The absolute configuration of the natural product was shown to be $(4 S, 5 S)$ by comparing its spectral and analytical data with the reported values.

\section{Introduction}

Cancer is at present the second most common cause of death, after cardiovascular diseases, and will become the primary cause in the next 10 to 20 years [1]. Traditional cancer therapies make use of chemotherapy at the maximum tolerated dose. This approach has generally considerable associated toxicity, often with limited success. Therefore, more universal, more effective, and less toxic therapeutic agents are desirable. Recently, inhibition of angiogenesis has been considered as a desirable pathway for preventing tumor growth and metastasis, primarily because of the low potential for toxicity or resistance
[2], as well as the potential for treating a broad spectrum of tumor types, arthritis, and psoriasis [3-8]. For this reason, angiogenesis inhibition has become an active area of pharmaceutical research, and over 40 such agents are currently undergoing clinical trials [9]. In particular, efforts have been focused on small-molecules based on inhibitors isolated from natural products that can block tumor angiogenesis $[10,11]$.

Recently, streptopyrrolidine (Figure 1), was isolated as an angiogenesis inhibitor from the fermentation broth of a marine 
Streptomyces sp. found in deep sea sediments [12]. The system is present in many biologically active compounds [13-21] and it could act as a versatile intermediate for the synthesis of a wide range of $\gamma$-amino acids as well as pyrrolidines [22,23]. The interesting chemical structure and potent anti-angiogenic activity at non-toxic threshold doses of streptopyrrolidine attracted our attention for developing a concise and efficient protocol for its synthesis in sizable amounts for further biological studies. To date, three syntheses $[15,24,25]$ of $(4 S, 5 S)$ streptopyrrolidine have been described, two of which $[15,24]$ were reported only as a synthetic intermediate prior to its isolation from the natural source.<smiles>O=C1CC(O)C(Cc2ccccc2)N1</smiles>

$(4 R, 5 R)$-streptopyrrolidine (1)<smiles>O=C1CC(O)[C@H](Cc2ccccc2)N1</smiles>

$(4 R, 5 S)$-streptopyrrolidine (3)

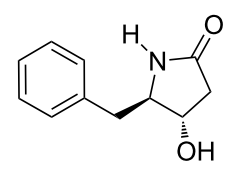

$(4 S, 5 R)$-streptopyrrolidine (2)<smiles>O=C1C[C@@H](O)[C@H](Cc2ccccc2)N1</smiles>

$(4 \mathrm{~S}, 5 \mathrm{~S})$-streptopyrrolidine (4)
Figure 1: Structures of $(4 R, 5 R)$-streptopyrrolidine (1), (4S,5R)-streptopyrrolidine (2) $(4 R, 5 S)$-streptopyrrolidine $(3)$ and $(4 S, 5 S)$-streptopyrrolidine (4).

\section{Results and Discussion}

In this paper, we report the syntheses of $(4 R, 5 R)$-streptopyrrolidine (1), $(4 S, 5 R)$-streptopyrrolidine (2), $(4 R, 5 S)$-streptopyrrolidine (3) and $(4 S, 5 S)$-streptopyrrolidine (4) in a concise and highly efficient manner via a highly stereoselective aldol type reaction and Lewis acid mediated lactamization as the key reactions. A retrosynthetic analysis for streptopyrrolidine is depicted in Scheme 1.

The synthesis was initiated from D-phenylalanine (7) which was converted to $N$-Boc-D-phenylalaninal (6) in $76 \%$ yield in<smiles>C=CC=CCC[C@H]1NC(=O)C[C@H]1O</smiles><smiles>N[C@@H](Cc1ccccc1)C(=O)O</smiles><smiles>CCOC(=O)CC(O)[C@H](N)Cc1ccccc1</smiles><smiles>CC(C)(C)OC(=O)C[C@H](C=O)Cc1ccccc1</smiles>

Scheme 1: Retrosynthetic analysis. three steps by a known protocol [26,27]. The aldehyde was treated with the lithium enolate of ethyl acetate [28,29] at -78 ${ }^{\circ} \mathrm{C}$ according to a modification of the procedure of Steulmann and Klostermeyer [30] to afford two diastereomers $\mathbf{8 a}$ and $\mathbf{8 b}$ in a 3:2 ratio (as determined by NMR) (Scheme 2).

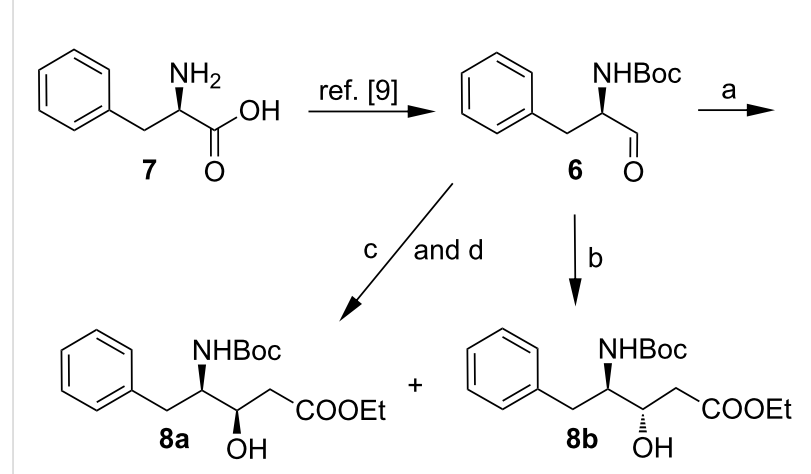

Scheme 2: Reagents and conditions: (a) LDA, EtOAc, THF, $-78^{\circ} \mathrm{C}$, $4 \mathrm{~h}, 80 \%(8 \mathbf{a}: 8 \mathbf{b}=3: 2)$; (b) $\mathrm{BF}_{3} \cdot \mathrm{OEt}_{2}$, (1-ethoxyvinyloxy)trimethylsilane, $\mathrm{CH}_{2} \mathrm{Cl}_{2},-78{ }^{\circ} \mathrm{C}, 2 \mathrm{~h}, 85 \%$ (exclusively $8 \mathrm{~b}$ ); (c) $\mathrm{SnCl}_{4}$, (1-ethoxyvinyloxy)trimethylsilane, $\mathrm{CH}_{2} \mathrm{Cl}_{2},-78{ }^{\circ} \mathrm{C}, 2 \mathrm{~h}, 70 \%(8 \mathbf{a}: 8 \mathbf{b}=$ $3: 7)$; (d) LDA, EtOAc, $\mathrm{ZnBr}_{2},-78{ }^{\circ} \mathrm{C}, 1 \mathrm{~h}, 82 \%,(\mathbf{8 a : 8 b}=95: 5)$.

Diastereomers $\mathbf{8 a}$ and $\mathbf{8 b}$ were easily separated by standard column chromatography on silica gel. The pioneering work on catalytic aldol reactions recently reported by Shibasaki [31] prompted us to investigate whether the ketene silyl acetal could be utilized for the aldol reaction in the presence of different Lewis acids to obtain a better selectivity particularly for $(R)$ tert-butyl (1-oxo-3-phenylpropan-2-yl)carbamate (6). To our surprise, when the reaction was carried out with the ketene silyl acetate at $-78{ }^{\circ} \mathrm{C}$, of the five Lewis acids investigated $\left(\mathrm{SnCl}_{4}\right.$, $\left.\mathrm{BF}_{3} \cdot \mathrm{OEt}_{2}, \mathrm{ZnI}_{2}, \mathrm{TiCl}_{4}, \mathrm{EtOAc} / \mathrm{LDA} / \mathrm{ZnBr}_{2}\right) \mathrm{BF}_{3} \cdot \mathrm{OEt}_{2}$ gave excellent selectivity (exclusively $\mathbf{8 b}$ ) (Table 1 ).

Following a modification of the Mosher method [32,33], the newly created stereogenic center in compound $\mathbf{8 b}$ bearing the hydroxyl group was assigned. The syntheses of both the $(S)$ and $(R)$-MTPA ester of $\mathbf{8 b}$ were achieved using MTPA acid with DCC as the coupling reagent. The chemical shifts of both the $(S)$ - and $(R)$-MTPA esters of $\mathbf{8 b}$ were assigned by ${ }^{1} \mathrm{H}$ NMR. From the equation given in Figure 2, the $\Delta \delta$ values were calculated for as many protons as possible. The carbon chain bearing protons showing $\Delta \delta$ negative values should be placed on the left hand side of the model (Figure 2) whilst that where $\Delta \delta$ has positive values should be placed on the right hand side. From this the center was found to have the $S$-configuration which thus establishes the absolute stereochemistry of $\mathbf{8 a}$. With the absolute stereochemistry of both the isomers known, we were interested to develop a protocol to have control over the selectivity to obtain exclusively $\mathbf{8 a}$. Stereoselective addition of the 
Table 1: Addition reaction under different conditions to obtain $\mathbf{8 a}$ and $\mathbf{8 b}$.

\begin{tabular}{lccccc} 
Entry & Reagent & Additive (Lewis Acid) & Time $(\mathrm{h})$ & Yield ${ }^{\mathrm{a}}(\%)$ & $\mathbf{8 a : 8 b}$ \\
\hline 1 & ketene silyl acetate & $\mathrm{SnCl}_{4}$ & 2 & 70 & $30: 70$ \\
2 & ketene silyl acetate & $\mathrm{BF}_{3} \cdot \mathrm{OEt}_{2}$ & 2 & 85 & $0: 100$ \\
3 & ketene silyl acetate & $\mathrm{TiCl}_{4}$ & 10 & 42 & $35: 65$ \\
4 & ketene silyl acetate & $\mathrm{ZnI}_{2}$ & 12 & 59 & $80: 20$ \\
5 & EtOAC/LDA & $\mathrm{ZnBr}_{2}$ & 1 & 82 & $95: 05$ \\
\hline
\end{tabular}

aisolated yield.

zinc enolate [34] of ethyl acetate to $N$-Boc-phenylalaninal (6) resulted in 8a as a 95:5 mixture of diastereomers (as determined by HPLC), which were easily separated by silica gel column chromatography. The stereochemical outcome in both the cases was explained by Crams' rule, i.e., for a non-chelation model, the $(R)$-aldehyde would give rise to the $(3 S, 4 R)$ isomer. Alternatively, in a chelation model, the metal imposes a syn relationship between the formyl and neighboring nitrogen functionality which would lead to the $(3 R, 4 R)$ isomer [35].

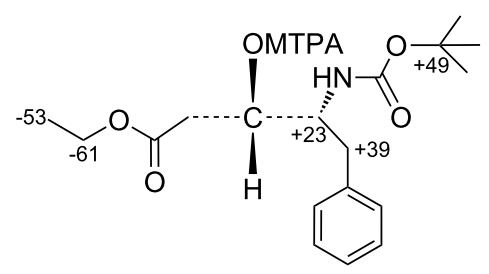

Figure 2: $\Delta \delta=\left(\delta_{\mathrm{S}}-\delta_{\mathrm{R}}\right) \times 10^{3}$ for $(S)$ - and $(R)$-MTPA esters of compound $\mathbf{8 b}$.

Deprotection of the Boc-group with TFA and $\mathrm{CH}_{2} \mathrm{Cl}_{2}$ was followed by evaporation and treatment of the crude product under different reaction conditions for lactamization (Table 2 is for lactamization of $\mathbf{8 a}$, almost similar yields were obtained for $\mathbf{8 b}$ ), afforded $(4 R, 5 R)$-streptopyrrolidine (1) and $(4 S, 5 R)$-streptopyrrolidine (2) in good to excellent overall yield (Scheme 3 ). From all of the conditions investigated, $\mathrm{Zr}\left(\mathrm{O}^{t} \mathrm{Bu}\right)_{4}$ in presence of 1-hydroxy-7-azabenzotriazole (HOAt) gave the best result [36], i.e., $82 \%$ yield over the two steps. The spectral and analytical data $\left\{[\alpha]_{\mathrm{D}}{ }^{25}+40.5(c 1.8, \mathrm{MeOH})\right.$; lit. $[15,25][\alpha]_{\mathrm{D}}{ }^{25}-43.5$ (c 1.0, MeOH) for its enantiomer\} of compound 1 [6] were in good agreement with the reported values for its enantiomer except the sign of the specific rotation, which confirmed the absolute configuration of the natural product as $(4 S, 5 S)$. To confirm further the absolute stereochemistry of natural streptopyrrolidine, $(4 R, 5 S)$-streptopyrrolidine (3) and (4S, 5S)-streptopyrrolidine (4) were prepared starting from L-phenylalanine. The spectral and analytical data of $(4 S, 5 S)$-streptopyrrolidine were in good agreement with natural streptopyrrolidine.<smiles>CCOC(=O)CC[C@H](O)[C@H]([18O])Cc1ccccc1</smiles>

$(4 R, 5 R)$-streptopyrrolidine (1)<smiles>CCOC(=O)CC[C@H](O)[C@H](N)Cc1ccccc1</smiles>
$(4 S, 5 R)$-streptopyrrolidine (2)

Scheme 3: Reagents and conditions: (a) (1) TFA, $\mathrm{CH}_{2} \mathrm{Cl}_{2}$, rt, $4 \mathrm{~h}$; (2) $\mathrm{Zr}\left(\mathrm{O}^{\mathrm{t} B u}\right)_{4}, \mathrm{HOAt}$, toluene, $60^{\circ} \mathrm{C}, 12 \mathrm{~h}, 82 \%$ over two steps.

\begin{tabular}{|c|c|c|c|c|c|}
\hline Entry & Reagent & Additive/ Solvent & Temp. $\left({ }^{\circ} \mathrm{C}\right)$ & Time $(\mathrm{h})$ & Yield $^{a}(\%)$ \\
\hline 1 & Py & none $/ \mathrm{CH}_{2} \mathrm{Cl}_{2}$ & 50 & 12 & 41 \\
\hline 2 & $\mathrm{Et}_{3} \mathrm{~N}$ & none $/ \mathrm{CH}_{2} \mathrm{Cl}_{2}$ & 50 & 12 & 46 \\
\hline 3 & Py & none/toluene & 100 & 6 & 55 \\
\hline 4 & $\mathrm{Et}_{3} \mathrm{~N}$ & none/toluene & 100 & 6 & 58 \\
\hline 5 & $\mathrm{KO}^{t} \mathrm{Bu}$ & HOAt/toluene & 60 & 6 & 42 \\
\hline 6 & $\mathrm{Zr}\left(\mathrm{O}^{t} \mathrm{Bu}\right)_{4}$ & HOAt/toluene & 60 & 6 & 82 \\
\hline
\end{tabular}

ayield over two steps. 


\section{Conclusion}

In conclusion, the total synthesis of all four possible isomers of streptopyrrolidine (1-4) has been achieved in $\approx 42 \%$ yield over 6 steps starting from either D- or L-phenylalanine which further confirmed the absolute configuration of natural streptopyrrolidine. Our protocol is highly flexible for the synthesis of all four possible isomers of streptopyrrolidine compared to previous reports.

\section{Experimental}

\section{General information}

All reactions were carried out under an inert atmosphere, unless otherwise stated. Solvents were dried and purified by standard methods prior to use. The progress of all reactions was monitored by TLC using glass plates pre-coated with silica gel 60 F254 with a thickness of $0.5 \mathrm{~mm}$. Column chromatography was performed on silica gel (60 mesh) with ethyl acetate and hexane the eluent. Optical rotations were measured with a Perkin Elmer P241 polarimeter and a JASCO DIP-360 digital polarimeter at $25^{\circ} \mathrm{C}$. IR spectra were recorded on a Perkin-Elmer FT-IR spectrometer. ${ }^{1} \mathrm{H}$ and ${ }^{13} \mathrm{C}$ NMR spectra were recorded on a Variant Gemini $200 \mathrm{MHz}$, Bruker Avance $300 \mathrm{MHz}$, or Varian Inova $500 \mathrm{MHz}$ spectrometer with TMS as an internal standard in $\mathrm{CDCl}_{3}, \mathrm{CD}_{3} \mathrm{OD}$ etc. Mass spectra were recorded on a Micromass VG-7070H for EI.

(3R,4R)-Ethyl-4-(tert-butoxycarbonylamino)-3-hydroxy-5phenylpentanoate $(8 \mathrm{a})$ and $(3 S, 4 R)$-ethyl-4-(tert-butoxycarbonylamino)-3-hydroxy-5-phenylpentanoate $(8 b)$ : To a stirred solution of the aldehyde $6(0.2 \mathrm{~g}, 0.8 \mathrm{mmol})$ in $\mathrm{CH}_{2} \mathrm{Cl}_{2}$ $(5 \mathrm{~mL})$ under a nitrogen atmosphere and cooled to $-78^{\circ} \mathrm{C}$, was added $1 \mathrm{M}$ solution of $\mathrm{SnCl}_{4}$ in $\mathrm{CH}_{2} \mathrm{Cl}_{2}(0.1 \mathrm{~mL}, 0.81 \mathrm{mmol})$ After $10 \mathrm{~min}$, (1-ethoxyvinyloxy)trimethylsilane (0.26 g, 1.6 mmol) was added at the same temperature. The reaction mixture was stirred for $1 \mathrm{~h}$ at $-78{ }^{\circ} \mathrm{C}$. After completion of the reaction (as determined by TLC), the reaction mixture was quenched with $1 \mathrm{~N} \mathrm{KOH}(3 \mathrm{~mL})$. The reaction mixture was extracted with ethyl acetate $(3 \times 25 \mathrm{~mL})$. The combined organic layers were washed with brine, dried over $\mathrm{Na}_{2} \mathrm{SO}_{4}$ and concentrated under reduced pressure to obtain pale yellow liquid which on purification by silica gel column chromatography afforded $\mathbf{8 a}$ and $\mathbf{8 b}$ in a ratio of 3:7 $(0.19 \mathrm{~g}, 70 \%)$ as colorless viscous liquid.

To a stirred solution of the aldehyde $6(0.1 \mathrm{~g}, 0.4 \mathrm{mmol})$ in $\mathrm{CH}_{2} \mathrm{Cl}_{2}(5 \mathrm{~mL})$ under a nitrogen atmosphere and cooled to -78 ${ }^{\circ} \mathrm{C}$, was added $\mathrm{BF}_{3} \cdot \mathrm{OEt}_{2}(0.03 \mathrm{~g}, 0.2 \mathrm{mmol})$. After $30 \mathrm{~min}$, (1-ethoxy vinyloxy)trimethylsilane $(0.26 \mathrm{~g}, 1.6 \mathrm{mmol})$ was added at the same temperature. The reaction mixture was stirred for $1 \mathrm{~h}$ at $-78^{\circ} \mathrm{C}$ when TLC showed completion of the reaction. The reaction mixture was quenched with saturated aqueous
$\mathrm{Na}_{2} \mathrm{CO}_{3}$, extracted with ethyl acetate $(3 \times 25 \mathrm{~mL})$. The combined organic layers were washed with brine, dried over $\mathrm{Na}_{2} \mathrm{SO}_{4}$ and concentrated under reduced pressure to give a pale yellow liquid. The crude product was purified by silica gel column chromatography with ethyl acetate and hexane (1:3) as eluent to yield $\mathbf{8 b}$ as a colorless viscous liquid as the sole product $(0.115 \mathrm{~g}, 85 \%)$.

A stirred solution of LDA (4.9 mL, $2 \mathrm{~N}, 9.8 \mathrm{mmol})$ in anhydrous THF $(10 \mathrm{~mL})$ was cooled to $-78{ }^{\circ} \mathrm{C}$ under nitrogen atmosphere. Ethyl acetate $(1.05 \mathrm{~mL}, 9.8 \mathrm{mmol})$ was then added followed by a $0{ }^{\circ} \mathrm{C}$ solution of anhydrous $\mathrm{ZnBr}_{2}(2.17 \mathrm{~g}, 9.8$ $\mathrm{mmol})$ in anhydrous THF $(5 \mathrm{~mL})$. A $-78^{\circ} \mathrm{C}$ solution of $N$-Bocprotected aldehyde $6(0.35 \mathrm{~g}, 1.41 \mathrm{mmol})$ in anhydrous THF (4 $\mathrm{mL}$ ) was added and the mixture stirred at $-78^{\circ} \mathrm{C}$ for $30 \mathrm{~min}$ then allowed to warm to room temperature. The reaction mixture was stirred at room temperature for $10 \mathrm{~h}$. After completion of the reaction (as determined by TLC), saturated $\mathrm{NH}_{4} \mathrm{Cl} /$ acetic acid $(9: 1)(20 \mathrm{~mL})$ was added to the reaction mixture which was then extracted with ethyl acetate $(3 \times 30 \mathrm{~mL})$. The combined organic layers were washed with brine $(2 \times 50 \mathrm{~mL})$, dried over $\mathrm{Na}_{2} \mathrm{SO}_{4}$, concentrated under reduced pressure, and the crude product purified by silica gel column chromatography to give 8a $(0.378 \mathrm{~g}, 82 \%)$ as a colorless viscous liquid. Analytical and spectral data of 8a: $[\alpha]_{\mathrm{D}}{ }^{25}+34.9(c 1.2, \mathrm{MeOH})$; IR $(\mathrm{KBr})$ : 3553, 3496, 3376, 2979, 2934, 1727, $1683 \mathrm{~cm}^{-1}$; ${ }^{1} \mathrm{H}$ NMR (300 $\left.\mathrm{MHz}, \mathrm{CDCl}_{3}\right): 7.28-7.20(\mathrm{~m}, 5 \mathrm{H}, \mathrm{Ar} H), 5.07$ (d, $J=9.6 \mathrm{~Hz}$, $1 \mathrm{H}, \mathrm{NH}), 4.16-4.09$ (q, $J=6.9,14.1 \mathrm{~Hz}, 2 \mathrm{H}, \mathrm{OCH}_{2} \mathrm{CH}_{3}$ ), 3.98 $(\mathrm{d}, J=9.6 \mathrm{~Hz}, 1 \mathrm{H}, \mathrm{CHOH}), 3.74(\mathrm{q}, J=8.1,16.2 \mathrm{~Hz}, 1 \mathrm{H}$, $\mathrm{C} H \mathrm{NH}), 2.91\left(\mathrm{~d}, J=7.5 \mathrm{~Hz}, 2 \mathrm{H}, \mathrm{PhCH}_{2}\right), 2.59$ (dd, $J=10.1$, $\left.16.8 \mathrm{~Hz}, 1 \mathrm{H}, \mathrm{CH}_{2}{ }_{2} \mathrm{COO}\right), 2.38(\mathrm{dd}, J=2.3,16.9 \mathrm{~Hz}, 1 \mathrm{H}$, $\left.\mathrm{CH}_{2} \mathrm{COO}\right), 1.41(\mathrm{~s}, 9 \mathrm{H}, t-\mathrm{Bu}), 1.23(\mathrm{t}, J=6.9 \mathrm{~Hz}, 3 \mathrm{H}$, $\left.\mathrm{OCH}_{2} \mathrm{CH}_{3}\right) ;{ }^{13} \mathrm{C} \mathrm{NMR}\left(75 \mathrm{MHz}, \mathrm{CDCl}_{3}\right): 173.4,155.8,138.1$, 129.3, 128.4, 126.3, 79.3, 67.0, 60.7, 55.3, 38.5, 29.6, 28.3, 14.0; ESI-MS: $m / z=338[\mathrm{M}+\mathrm{H}]^{+}$; ESI-HRMS: Calcd. for $\mathrm{C}_{18} \mathrm{H}_{27} \mathrm{NNaO}_{5}, 360.1781$; found: 360.1789 . Analytical and spectral data of 8b: $[\alpha]_{\mathrm{D}}{ }^{25}+11.6(c 0.6, \mathrm{MeOH})$; IR $(\mathrm{KBr})$ : 3354, 2982, 2936, 1735, $1684 \mathrm{~cm}^{-1}$; ${ }^{1} \mathrm{H}$ NMR (300 MHz, $\left.\mathrm{CDCl}_{3}\right) 7.31-7.20(\mathrm{~m}, 5 \mathrm{H}, \mathrm{Ar} H), 4.61(\mathrm{~d}, J=8.4 \mathrm{~Hz}, 1 \mathrm{H}, \mathrm{NH})$, $4.20-4.13$ (q, $\left.J=7.1,14.1 \mathrm{~Hz}, 2 \mathrm{H}, \mathrm{OCH}_{2} \mathrm{CH}_{3}\right), 3.99-3.86$ (m, $2 \mathrm{H}, \mathrm{CHOH}, \mathrm{C} H \mathrm{NH}), 2.96$ (dd, $\left.J=3.8,13.7 \mathrm{~Hz}, 1 \mathrm{H}, \mathrm{PhCH}_{2}^{\prime}\right)$, $2.82\left(\mathrm{~m}, 1 \mathrm{H}, \mathrm{PhCH}_{2}\right), 2.64-2.45\left(\mathrm{~m}, 2 \mathrm{H}, \mathrm{CH}_{2} \mathrm{COO}\right), 1.34$ (s, $9 \mathrm{H}, t-\mathrm{Bu}), 1.25\left(\mathrm{t}, J=7.1 \mathrm{~Hz}, 3 \mathrm{H}, \mathrm{OCH}_{2} \mathrm{CH}_{3}\right) ;{ }^{13} \mathrm{C} \mathrm{NMR}(75$ $\left.\mathrm{MHz}, \mathrm{CDCl}_{3}\right)$ : 172.9, 155.7, 137.6, 129.4, 128.4, 126.4, 79.6, 70.1, 60.8, 55.1, 38.2, 35.8, 28.2, 14.1; ESI-MS: $m / z=360[\mathrm{M}$ $+\mathrm{Na}]^{+}$; ESI-HRMS: Calcd. for $\mathrm{C}_{18} \mathrm{H}_{27} \mathrm{NNaO}_{5}, 360.1781$; found: 360.1794 .

$(4 R, 5 R)$-streptopyrrolidine $(1),(4 S, 5 R)$-streptopyrrolidine (2), $(4 R, 5 S)$-streptopyrrolidine $(3),(4 S, 5 S)$-streptopyrrolidine (4): A solution of $\mathrm{TFA} / \mathrm{H}_{2} \mathrm{O}(2.8 \mathrm{~mL}, 0.28 \mathrm{~mL})$ 
was added to $8 \mathbf{a}$ or $\mathbf{8 b}(200 \mathrm{mg}, 0.59 \mathrm{mmol})$ and the resulting mixture stirred at room temperature for $3 \mathrm{~h}$. After completion of the reaction (as determined by TLC), the solvent was evaporated under reduced pressure and the resulting reddish oil dissolved in ethyl acetate $(10 \mathrm{~mL})$. The organic phase was washed with $\mathrm{NaHCO}_{3}$, dried over $\mathrm{Na}_{2} \mathrm{SO}_{4}$ and concentrated under reduced pressure. The red oil so obtained was dissolved in toluene. $\mathrm{Zr}\left(\mathrm{O}^{t} \mathrm{Bu}\right)_{4}(22 \mathrm{mg}, 0.059 \mathrm{mmol})$ followed by HOAt (160 $\mathrm{mg}, 0.11 \mathrm{mmol}$ ) were added and the reaction mixture allowed to stir at $60{ }^{\circ} \mathrm{C}$ for $12 \mathrm{~h}$. After completion of the reaction (as determined by TLC), the toluene was evaporated under reduced pressure. Water was added and the reaction mixture extracted with $\mathrm{CH}_{2} \mathrm{Cl}_{2}(3 \times 20 \mathrm{~mL})$. The combined organic layers were dried over $\mathrm{Na}_{2} \mathrm{SO}_{4}$ and concentrated under reduced pressure to give a brown liquid which on purification by silica gel column chromatography furnished $\mathbf{1}$ (98 $\mathrm{mg}, 82 \%$ ) as a white sticky solid. Analytical and spectral data of 1: $[\alpha]_{\mathrm{D}}{ }^{25}$ +40.5 (c 1.8, MeOH); IR (KBr): 3452, 3234, 2924, $1690 \mathrm{~cm}^{-1}$; ${ }^{1} \mathrm{H}$ NMR (500 MHz, DMSO-d 6 ): $7.51(\mathrm{~s}, 1 \mathrm{H}, \mathrm{NH}), 7.27(\mathrm{~m}$, $4 \mathrm{H}, \mathrm{ArH}), 7.17$ (m, 1H, ArH), $5.14(\mathrm{~d}, J=4.1 \mathrm{~Hz}, 1 \mathrm{H}, \mathrm{OH})$, $4.10(\mathrm{~s}, 1 \mathrm{H}, \mathrm{H}-4), 3.67$ (q, $J=5.6 \mathrm{~Hz}, 1 \mathrm{H}, \mathrm{H}-5), 2.96$ (dd, $J=$ 7.9, $\left.13.4 \mathrm{~Hz}, 1 \mathrm{H}, \mathrm{NCHCH}_{2}^{\prime}\right), 2.65(\mathrm{dd}, J=6.2,13.4 \mathrm{~Hz}, 1 \mathrm{H}$, $\left.\mathrm{NCHCH}_{2}\right), 2.38\left(\mathrm{dd}, J=6.1,16.4 \mathrm{~Hz}, 1 \mathrm{H}, \mathrm{H}-3{ }^{\prime}\right), 1.96$ (dd, $J=$ 2.6, $16.4 \mathrm{~Hz}, 1 \mathrm{H}, \mathrm{H}-3) ;{ }^{13} \mathrm{C}$ NMR (75 MHz, DMSO- $\left.d_{6}\right): 174.8$, 138.6, 129.2, 128.1, 125.9, 66.9, 60.0, 40.8, 34.5; EIMS: 192 $[\mathrm{M}+\mathrm{H}]^{+}$; ESI-HRMS: Calcd. for $\mathrm{C}_{11} \mathrm{H}_{14} \mathrm{NO}_{2}, 192.1019$; found: 192.1026. Analytical and spectral data of $2:[\alpha]_{\mathrm{D}}{ }^{25}-13.2(\mathrm{c}$ 1.6, MeOH); IR (neat): 3384, 2925, 2855, $1679 \mathrm{~cm}^{-1} ;{ }^{1} \mathrm{H}$ NMR (500 MHz, DMSO- $\left.d_{6}\right): 7.68(\mathrm{~s}, 1 \mathrm{H}, \mathrm{NH}), 7.29-7.21(\mathrm{~m}, 5 \mathrm{H}$, ArH), 5.13 (d, J=3.9 Hz, 1H, OH), 3.95 (s, 1H, H-4), 3.51 (t, $J$ $=6.8 \mathrm{~Hz}, 1 \mathrm{H}, \mathrm{H}-5), 2.69\left(\mathrm{~m}, 2 \mathrm{H}, \mathrm{NCHCH}_{2}\right), 2.27(\mathrm{dd}, J=6.8$, $17.5 \mathrm{~Hz}, 1 \mathrm{H}, \mathrm{H}-3$ '), 1.80 (dd, $J=1.9,16.5 \mathrm{~Hz}, 1 \mathrm{H}, \mathrm{H}-3) ;{ }^{13} \mathrm{C}$ NMR (75 MHz, DMSO-d $d_{6}$ ): 174.8, 137.6, 129.4, 128.4, 126.2, 69.3, 63.4, 40.0, 38.9; ESI-HRMS: Calcd. for $\mathrm{C}_{11} \mathrm{H}_{14} \mathrm{NO}_{2}$, 192.1019; found: 192.1024. Analytical and spectral data of 3: $[\alpha]_{\mathrm{D}}{ }^{25}+12.6(c 1.2, \mathrm{MeOH})$; IR $(\mathrm{KBr}): 3462,2928,1784$, 1730,1450, 1147, 1069, $744.8 \mathrm{~cm}^{-1}$; ${ }^{1} \mathrm{H}$ NMR $(300 \mathrm{MHz}$, DMSO-d $_{6}$ ): $\delta 7.70(\mathrm{~s}, 1 \mathrm{H}, \mathrm{NH}), 7.18-7.32(\mathrm{~m}, 5 \mathrm{H}, \mathrm{ArH}), 5.14$ (d, $J=3.7 \mathrm{~Hz}, 1 \mathrm{H}, \mathrm{OH}), 3.95$ (s, 1H, H-4), 3.51 (q, $J=6.0,13.2$ $\mathrm{Hz}, 1 \mathrm{H}, \mathrm{H}-5), 2.67$ (d, $\left.J=2.2 \mathrm{~Hz}, 2 \mathrm{H}, \mathrm{NCHCH}_{2}\right), 2.27$ (dd, $1 \mathrm{H}$, $\left.J=6.0,16.6 \mathrm{~Hz}, 1 \mathrm{H}, \mathrm{H}-3^{\prime}\right), 1.82(\mathrm{dd}, J=2.2,16.6 \mathrm{~Hz}, 1 \mathrm{H}$, $\mathrm{H}-3) ;{ }^{13} \mathrm{C}$ NMR $\left(75 \mathrm{MHz}, \mathrm{DMSO}-d_{6}\right): 174.8,137.6,129.4$, 128.2, 126.0, 69.2, 63.4, 39.9, 39.1; ESI-HRMS: Calcd. for $\mathrm{C}_{11} \mathrm{H}_{14} \mathrm{NO}_{2}, 192.1019$; found: 192.1026. Analytical and spectral data of 4: $[\alpha]_{\mathrm{D}}{ }^{25}-41.2(c 1.0, \mathrm{MeOH})$; IR (KBr): 3451 , $1785,1453,1185,1147,1058,786,698 \mathrm{~cm}^{-1} ;{ }^{1} \mathrm{H}$ NMR $(300$ $\left.\mathrm{MHz}, \mathrm{DMSO}-d_{6}\right): \delta 7.55(\mathrm{~s}, 1 \mathrm{H}, \mathrm{NH}), 7.08-7.37$ (m, 5H, ArH), $5.18(\mathrm{~d}, J=3.8 \mathrm{~Hz}, 1 \mathrm{H}, \mathrm{OH}), 4.09(\mathrm{~s}, 1 \mathrm{H}, \mathrm{H}-4), 3.67$ (s, $1 \mathrm{H}$, $\mathrm{H}-5), 2.96\left(\mathrm{dd}, J=7.1,12.6 \mathrm{~Hz}, 1 \mathrm{H}, \mathrm{NCHCH}_{2}{ }^{\prime}\right), 2.66$ (dd, $J=$ $\left.8.0,16.9 \mathrm{~Hz}, 1 \mathrm{H}, \mathrm{NCHCH}_{2}\right), 2.38(\mathrm{dd}, J=6.1,15.6 \mathrm{~Hz}, 1 \mathrm{H}$, H-3'), 1.99 (dd, $J=3.0,15.6 \mathrm{~Hz}, 1 \mathrm{H}, \mathrm{H}-3) ;{ }^{13} \mathrm{C}$ NMR $(75 \mathrm{MHz}$,
DMSO- $\left.d_{6}\right): 174.9,138.6,129.2,128.2,126.8,66.9,60.1,40.8$, 34.5; ESI-HRMS: Calcd. for $\mathrm{C}_{11} \mathrm{H}_{14} \mathrm{NO}_{2}, 192.1019$; found: 192.1026 .

\section{Supporting Information}

Supporting Information features ${ }^{1} \mathrm{H}$ and ${ }^{13} \mathrm{C}$ NMR spectra of all intermediates.

\section{Supporting Information File 1}

${ }^{1} \mathrm{H}$ and ${ }^{13} \mathrm{C}$ NMR spectra of all intermediates.

[http://www.beilstein-journals.org/bjoc/content/ supplementary/1860-5397-7-6-S1.pdf]

\section{Acknowledgements}

B.T. and P.P.D. thank UGC and CSIR, New Delhi, India, respectively, for the financial assistance in the form of fellowships.

\section{References}

1. Cancer Facts \& Figures. http://www.cancer.org/Research/CancerFactsFigures/CancerFactsFigu res/index (accessed Sept 1, 2010).

2. Folkman, J. Nat. Med. 1995, 1, 27-30. doi:10.1038/nm0195-27

3. Folkman, J. J. Natl. Cancer Inst. 1990, 82, 4-6. doi:10.1093/jnci/82.1.4

4. Folkman, J. Breast Cancer Res. Treat. 1995, 36, 109-118. doi:10.1007/BF00666033

5. Hanahan, D.; Folkman, J. Cell 1996, 86, 353-364. doi:10.1016/S0092-8674(00)80108-7

6. Ferrara, N.; Alitalo, K. Nat. Med. 1999, 5, 1359-1364. doi:10.1038/70928

7. Yancopouls, G. D.; Davis, S.; Gale, N. W.; Rudge, J. S.; Wiegand, S. J.; Holash, J. Nature 2000, 407, 242-248. doi:10.1038/35025215

8. Carmeliet, P.; Jain, R. K. Nature 2000, 407, 249-257. doi: $10.1038 / 35025220$

9. Clinical Trials Home Page - National Cancer Institute. http://www.cancer.gov/clinicaltrials (accessed Sept 1, 2010). (See for information on current cancer clinical trials.)

10. McDermott, L. A.; Higgins, B.; Simcox, M.; Luk, K.-C.; Nevins, T.; Kolinsky, K.; Smith, M.; Yang, H.; Li, J. K.; Chen, Y.; Ke, J.; Mallalieu, N.; Egan, T.; Kolis, S.; Railkar, A.; Gerber, L.; Liu, J.-J.; Konzelmann, F.; Zhang, Z.; Flynn, T.; Morales, O.; Chen, Y. Bioorg. Med. Chem. Lett. 2006, 16, 1950-1953. doi:10.1016/j.bmcl.2005.12.092

11. Eskens, F. A. L. M.; Verweij, J. Eur. J. Cancer 2006, 42, 3127-3139. doi:10.1016/j.ejca.2006.09.015

12. Shin, H. J.; Kim, T. S.; Lee, H.-S.; Park, J. Y.; Choi, I.-K.; Kwon, H. J. Phytochemistry 2008, 69, 2363-2366. doi:10.1016/j.phytochem.2008.05.020

13. Vedejs, E.; Campbell, J. B., Jr.; Gadwood, R. C.; Rodgers, J. D.; Spear, K. L.; Watanabe, Y. J. Org. Chem. 1982, 47, 1534-1546. doi:10.1021/jo00347a034

14. Midland, M. M.; Afonso, M. M. J. Am. Chem. Soc. 1989, 111, 4368-4371. doi:10.1021/ja00194a033 
15. Poncet, J.; Jouin, P.; Castro, B.; Nicolas, J.; Boutar, M.; Gaudemer, A. J. Chem. Soc., Perkin Trans. 1 1990, 611-616. doi:10.1039/P19900000611

16. Ohta, T.; Shiokawa, S.; Sakamoto, R.; Nozoe, S. Tetrahedron Lett. 1990, 31, 7329-7332. doi:10.1016/S0040-4039(00)88557-3

17. Koot, W.-J.; van Ginkel, R.; Kranenburg, M.; Hiemstra, H.; Louwrier, S.; Moolenaar, M. J.; Speckamp, W. M. Tetrahedron Lett. 1991, 32, 401-404. doi:10.1016/S0040-4039(00)92639-X

18. Rinehart, K. L.; Sakai, R.; Kishore, V.; Sullins, D. W.; Li, K.-M. J. Org. Chem. 1992, 57, 3007-3013. doi:10.1021/jo00037a012

19. Galeotti, N.; Poncet, J.; Chiche, L.; Jouin, P. J. Org. Chem. 1993, 58, 5370-5376. doi:10.1021/jo00072a018

20. Reddy, G. V.; Rao, G. V.; lyengar, D. S. Tetrahedron Lett. 1999, 40, 775-776. doi:10.1016/S0040-4039(98)02409-5

21. Lennartz, M.; Sadakane, M.; Steckhan, E. Tetrahedron 1999, 55, 14407-14420. doi:10.1016/S0040-4020(99)00904-7

22. Huang, P. G.; Zheng, X.; Wang, S. L.; Ye, J. L.; Jin, L. R.; Chen, Z. Tetrahedron: Asymmetry 1999, 10, 3309-3317. doi:10.1016/S0957-4166(99)00321-3

23. Park, T. H.; Paik, S.; Lee, S. H. Bull. Korean Chem. Soc. 2003, 24 , 1227-1228.

24. Kondekar, N. B.; Kandula, S. R. V.; Kumar, P. Tetrahedron Lett. 2004, 45, 5477-5479. doi:10.1016/j.tetlet.2004.05.057

25. Xiang, S.-H.; Yuan, H.-Q.; Huang, P.-Q. Tetrahedron: Asymmetry 2009, 20, 2021-2026. doi:10.1016/j.tetasy.2009.08.018

26. McKennon, M. J.; Meyers, A. I.; Drauz, K.; Schwarm, M. J. Org. Chem. 1993, 58, 3568-3571. doi:10.1021/jo00065a020

27. Myers, A. G.; Zhong, B.; Movassaghi, M.; Kung, D. W.; Lanman, B. A.; Kwon, S. Tetrahedron Lett. 2000, 41, 1359-1362. doi:10.1016/S0040-4039(99)02293-5

28. Rich, D. H.; Sun, E. T.; Boparia, A. S. J. Org. Chem. 1978, 43, 3624-3626. doi:10.1021/jo00412a053

29. McConnel, R. M.; Frizzell, D.; Camp, A.; Evans, A.; Jones, W.; Cagle, C. J. Med. Chem. 1991, 34, 2298-2300. doi:10.1021/jm00111a054

30. Steulmann, R.; Klostermeyer, H. Justus Liebigs Ann. Chem. 1975, 2245-2247

31. Oisaki, K.; Suto, Y.; Kanai, M.; Shibasaki, M. J. Am. Chem. Soc. 2003, 125, 5644-5645. doi:10.1021/ja034993n

32. Ohtani, I.; Kusumi, J.; Kashman, Y.; Kakisawa, H. J. Am. Chem. Soc. 1991, 113, 4092-4096. doi:10.1021/ja00011a006

33. Yoshida, W. Y.; Bryan, P. J.; Baker, B. J.; McClintock, J. B. J. Org. Chem. 1995, 60, 780-782. doi:10.1021/jo00108a057

34. Dell'Agli, M.; Parapini, S.; Galli, G.; Vaiana, N.; Taramelli, D.; Sparatore, A.; Liu, P.; Dunn, B. M.; Bosisio, E.; Romeo, S. J. Med. Chem. 2006, 49, 7440-7449. doi:10.1021/jm061033d

35. Danishefsky, S.; Kobayashi, S.; Kerwin, J. F., Jr. J. Org. Chem. 1982, 47, 1981-1983. doi:10.1021/jo00349a037 And references therein.

36. Han, C.; Lee, J. P.; Lobkovsky, E.; Porco, J. A., Jr. J. Am. Chem. Soc. 2005, 127, 10039-10044. doi:10.1021/ja0527976

\section{License and Terms}

This is an Open Access article under the terms of the Creative Commons Attribution License

(http://creativecommons.org/licenses/by/2.0), which permits unrestricted use, distribution, and reproduction in any medium, provided the original work is properly cited.

The license is subject to the Beilstein Journal of Organic Chemistry terms and conditions:

(http://www.beilstein-journals.org/bjoc)

The definitive version of this article is the electronic one which can be found at:

doi:10.3762/bjoc.7.6 